\title{
A PARAMETRIC REPRESENTATION OF RULED SURFACES
}

\author{
ELENA PROUSALIDOU, SEAN HANNA \\ University College London, United Kingdom
}

\begin{abstract}
This paper proposes a simple parametric system to generate an almost complete set of ruled surfaces that may be used to describe building geometry. The major classes of regular, named ruled surfaces can be generated from a limited set of curves. Each of these is shown to be reducible to a transformation of a single standard curve, a helix, and therefore represented by a limited set of six parameters. Six extra parameters can position each surface on a global coordinate system. The representation is designed to be flexible enough to represent both planar and curvilinear forms, producing a description of form from minimal data.
\end{abstract}

\section{Introduction}

Generative digital techniques attempt to exploit the power of information technology as a direct tool to represent and generate architectural forms. Demonstrative approaches in this new field of design techniques are parametric and evolutionary design, often used in combination. Their common feature is the design of the end product as an instance of a class of possible solutions. Another similarity they share is the description of the product by a series of parameters that take on different values. These values can either be input by the designer, in the first case, or be evolved by a program, in the latter. Parametric and generative representations of buildings, whether based on orthogonal (March and Steadman 1971; Rosenman and Gero 1999; Steadman and Waddoups 2000; Steadman 2001) or curvilinear (DeCOi 2000) geometry, are powerful by virtue of their ability to capture a high degree of variation in a few numerical values.

This paper proposes a parametric representation of ruled surfaces that uses a minimal set of variables to represent a wide variety of surfaces, including those most commonly used in architecture. A serial composition of the surfaces can be applied in the representation of building geometry. 


\subsection{ARCHITECTURAL STRUCTURES AS NUMERICAL SYSTEMS}

Configuration of built forms as represented by Lionel March in his isomorphic Boolean approach (March 1972) and Philip Steadman in his archetypal building (Steadman 1998) are indicative early attempts to represent architecture using a numerical description of form. Both use binary encoding that corresponds to a matrix of cuboids. Although they do achieve a compact representation of rectangular forms, the underlying relations of the building elements are restricted to proximity and vertical/horizontal packing.

\subsection{REPRESENTATIONS IN EVOLUTIONARY DESIGN}

Representation of form in strings is widely applied in Evolutionary Design techniques where the generative process operates with various parameters encoded into string-like structures.

Approaches focused on orthogonal spatial configurations, such as the learning approach developed by John Gero and the hierarchical growth approach developed by Mike Rosenman (Rosenman and Gero 1999) or the procedure proposed by Jackson (Jackson 2002), use genes to represent simple design actions as production rules or drawing instructions which when executed result in shapes or produce parts of design solutions (Bentley 1999).

Representations of 3dimensional form are often based on modular assemblies of primitive forms, such as the Reptile system proposed by Frazer (Frazer 1995), 'clipped stretched cuboids' developed by Peter Bentley and Jonathan Wakefield (Bentley 1999a), Form Grow by Stephen Todd and William Latham (1999) or the production of objects represented through the insertion of spheres at the isospatial grid as proposed by Paul Coates, Terence Broughton and Helen Jackson (Coates Broughton and Jackson 1999).

While these modules are quite simple, a parametric description of form may be created that is flexible enough to represent complex curves and surfaces. This is aptly demonstrated by Burry's extended application of parametric techniques on the analysis of the ruled surfaces used by Antonio Gaudi. He applies parametric design software at the Sagrada Familia with the intention "to remodel and resolve the use of these surfaces into a measurable interpretation that can be used to advance the building work", a form of rationalisation useful in design (Burry et al. 2001).

The proposed model incorporates Burry's work, and presents a simple surface description that may be used in evolutionary design and other parametric approaches. It can be evaluated by the numbers of parameters needed and the range of forms it is capable of representing. 


\section{Ruled surfaces}

A ruled surface can be generated by the motion of a line in space, similar to the way a curve can be generated by the motion of a point. A 3D surface is called ruled if through each of its points passes at least one line that lies entirely on that surface.

Simple to define and construct at a local level, ruled surfaces are able to describe high levels of form complexity, especially by their intersections when assembled (Burry 2003). Ruled surfaces have been extensively applied to architecture, with their potential exploited by new technological means. The Paramorph (DeCOi 2000) is an example.

\subsection{ARCHITECTURAL STRUCTURES}

Traditionally the plane, cylindrical and conical surfaces have been of the broadest use in architecture, and their familiarity often obscures their definition as ruled. The hyperboloid and the hyperbolic paraboloid are the most typical examples of more complex ruled surfaces, followed by the helicoid and, recently, the moebius strip. Construction of new structural systems using hyperboloids and hyperbolic paraboloids was first accomplished by Vladimir Shukhov, at the end of 19th century (English 2005). The doubly-curved surfaces were formed of a lattice of straight iron bars. Gaudi extended the application of ruled surfaces by using three surfaces that could be constructed at his time: hyperbolic paraboloid, helicoid and hyperboloid, all generated through straight lines. The entire design of the nave in the Sagrada Familia is an assembly of these three geometries (Burry 1993). These forms were extensively used by Felix Candela, and Le Corbusier's attraction to them is demonstrated by Philips Pavillion.

\subsection{SPECIAL FEATURES}

The most important feature of ruled surfaces in respect to design is their simplicity of construction relative to their striking shape. Burry (1993) emphasizes their ability to facilitate construction: "Any of these surfaces can be simply enough described as the points of origin and termination of an appropriate number of straight lines." Furthermore, the components of a larger whole can be carved independently off-site and then be assembled to form the final composition.

\section{Development of the parametric representation}

The most familiar way of constructing the surfaces in CAD packages is using lines that join corresponding points between two algebraic curves. In mathematical terms, however, the most common way of describing the 
surfaces is using a straight line moving along a curve with the direction given by another curve.

A surface is called a ruled surface, if it has a $\mathrm{C}^{2}$-parametrization of the following kind (Kühnel 2002):

$$
\mathrm{f}(\mathrm{u}, \mathrm{v})=\mathrm{b}(\mathrm{u})+\mathrm{v} \mathrm{X}(\mathrm{u})
$$

where $\mathrm{b}$ is a (differentiable, but not necessarily regular) curve and $\mathrm{X}$ is a vector field along $b$ which vanishes nowhere.

The v-lines (with constant $u$ ) are Euclidean lines in space. They are called generators or rulings of the surface and can be intuitively understood as the various positions of the generating line $\mathrm{L}$, the movement of which depends on one parameter.

The construction of a surface requires two parameterised curves, i.e. the base curve and the director curve. The base curve b (or directrix) of the surface is the curve along which runs the straight line. The line extends in the direction given by the director curve $\mathrm{d}$, which may be visualised as a vector field (a given sequence of unit vectors that varies continuously) on $b$. Mathematical equations for the two curves can be expressed as position vectors.

The parametric function of the helix expressed in vector form is:

$$
[\operatorname{acos}(\mathrm{t}), \mathrm{b} \sin (\mathrm{t}), \mathrm{ct}]
$$

Simple transformations of the helix result in a set of the curves needed to generate ruled surfaces. If the $\mathrm{z}$ vector component is suppressed the curve is degenerated to a circle. If the $\mathrm{x}$ vector component is suppressed the curve is degenerated to the parametric sine function. If the $\mathrm{x}$ and $\mathrm{y}$ vector components are suppressed the curve is degenerated to a line.

\begin{tabular}{|c|c|c|}
\hline & HELIX & {$[\operatorname{acos}(\mathrm{t}), \mathrm{bsin}(\mathrm{t}), \mathrm{ct}]$} \\
\hline & CIRCLE & {$[\mathrm{a} \cos (\mathrm{t}), \mathrm{b} \sin (\mathrm{t}), 0]$} \\
\hline & SINE & {$[0, \mathrm{~b} \sin (\mathrm{t}), \mathrm{ct}]$} \\
\hline & LINE & {$[0,0, \mathrm{ct}]$} \\
\hline
\end{tabular}

TABLE 1. Curves represented as Vectors of trigonometric functions. 


\begin{tabular}{|c|c|c|c|c|}
\hline & LINE & CIRCLE & HELIX & SINE \\
\hline \multicolumn{5}{|l|}{ LINE } \\
\hline \multicolumn{5}{|l|}{ CIRCLE } \\
\hline \multicolumn{5}{|l|}{ HELIX } \\
\hline \multicolumn{5}{|l|}{ SINE } \\
\hline \multicolumn{5}{|l|}{ LINE } \\
\hline \multicolumn{5}{|l|}{ CIRCLE } \\
\hline \multicolumn{5}{|l|}{ HELIX } \\
\hline \multicolumn{5}{|l|}{ SINE } \\
\hline \multicolumn{5}{|l|}{ LINE } \\
\hline \multicolumn{5}{|l|}{ CIRCLE } \\
\hline \multicolumn{5}{|l|}{ HELIX } \\
\hline SINE & & & & \\
\hline
\end{tabular}

TABLE 2. Ruled Surfaces 
The combination of these four curves as base and director respectively generates sixteen types of ruled surfaces. The rotation of one curve around the axes generates thirty two extra surfaces (sixteen for each rotation). Each surface of a structure can be expressed using six parameters: three for the base $\left(a_{b}, b_{b}, c_{b}\right)$ and three for the director curve $\left(a_{d}, b_{d}, c_{d}\right)$.

The plane and other surfaces require two curves along different axes. If the base curve is defined as mentioned above, the director curve should be defined either as

$\left[b_{d} \sin (t), c_{d} t, a_{d} \cos (t)\right]$

or

$\left[c_{d} t, a_{d} \cos (t), b_{d} \sin (t)\right]$

The procedure that was followed was the addition of an extra value in the $\mathrm{x}$ component of the director vector so that it becomes

$\left[a_{d} \cos (t)+d_{d} t, b_{d} \sin (t), c_{d} t\right]$

where parameter $d_{d}$ is applied for rotating one curve before generating the surface.

The surfaces generated in this way are presented in Table 2. The base curve is constantly oriented along the $Z$ axis while the director curve is oriented along axes $\mathrm{X}, \mathrm{Y}$ and $\mathrm{Z}$. Among these forty eight surfaces the plane, the cone, the cylinder, the hyperboloid and the helicoid are included, in addition to the commonly used sinusoidal surface.

When all $\mathrm{n}$ surfaces that form an architectural composition are indexed, a $13 \times \mathrm{n}$ matrix can represent a building.

$$
\left[\begin{array}{ccccccccccccc}
a_{b 1} & b_{b 1} & c_{b 1} & a_{d 1} & b_{d 1} & c_{d 1} & d_{d 1} & t_{x 1} & t_{y 1} & t_{z 1} & r_{x 1} & r_{y 1} & r_{z 1} \\
a_{b 2} & b_{b 2} & c_{b 2} & a_{d 2} & b_{d 2} & c_{d 2} & d_{d 2} & t_{x 2} & t_{y 2} & t_{z 2} & r_{x 2} & r_{y 2} & r_{z 2} \\
a_{b 3} & b_{b 3} & c_{b 3} & a_{d 3} & b_{d 3} & c_{d 3} & d_{d 3} & t_{x 3} & t_{y 3} & t_{z 3} & r_{x 3} & r_{y 3} & r_{z 3} \\
& & & & & & \ldots & & & & & & \\
& & & & & & \ldots & & & & & & \\
a_{b n} & b_{b n} & c_{b n} & a_{d n} & b_{d n} & c_{d n} & d_{d n} & t_{x n} & t_{y n} & t_{z n} & r_{x n} & r_{y n} & r_{z n}
\end{array}\right]
$$

Where:

$\alpha_{b}, b_{b}, c_{b}$ : parameters of base curve [ $\left.a \cos (t), b \sin (t), c t\right]$ and

$\alpha_{d}, b_{d}, c_{d}, d_{d}:$ parameters of director curve $[\operatorname{acos}(t)+d t, b \sin (t), c t]$

$t_{x}$ : translation of surface around $X$ axis

$t_{y}$ : translation of surface around $\mathrm{Y}$ axis 
$\mathrm{t}_{\mathrm{z}}$ : translation of surface around $\mathrm{Z}$ axis

$r_{x}$ : rotation of surface around $Z$ axis

$r_{y}$ : rotation of surface around $Z$ axis

$r_{z}$ : rotation of surface around $Z$ axis

\subsection{HYPERBOLIC PARABOLOID}

The above strategy represents many of the regular types of ruled surfaces, but the construction of the commonly used hyperbolic paraboloid requires the use of another type of curve, the parabola, which can not be produced by a linear transformation of the helix. Its function is expressed by the position vector

$$
\left[\mathrm{t}, 0, \mathrm{t}^{2}\right]
$$

and thus requires one additional, quadratic parameter $e_{d}$. The program was modified by adjusting the general function for the director curve:

$$
\left[a_{d} \cos (t)+d_{d} t, b_{d} \sin (t), c_{d} t+e_{d} t^{2}\right]
$$

To represent this extra class of surfaces this single extra parameter was added to the vector's components.

\section{Applying the representation}

The representation was implemented in a Processing program created to draw multiple surfaces. This receives a series of parameters for each surface as input by the user and draws an assembly of surfaces that represent the building.

Specifically, for each surface the program receives the parameters $a_{\mathrm{b}}, b_{\mathrm{b}}, c_{\mathrm{b}}$ and $a_{\mathrm{d}}, b_{\mathrm{d}}, c_{\mathrm{d}}, d_{\mathrm{d}}$ as input and executes the algorithm as follows:

At each step:

1. increment parameter $t$ by a constant number

2. calculate base and director curve vectors for value $t$ and draw a point with coordinates defined by each vector

3. join consecutive points to draw two curves

4. calculate slope of director curve by subtracting successive values for each vector component.

5. normalise slope vector

6. draw a line of fixed length starting from the point on the base curve and has its direction defined by the slope of the director curve, i.e a ruling.

This iterative process is repeated for every point of the base curve. A new line is generated for each value of parameter $t$. The assembly of those lines compose the surface. In order to draw the assembly of many surfaces as a unified whole the code was modified to include the surface as a class, so that 
multiple instances can be created simultaneously. As long as the values of the parameters for each surface vary, the instances created display a wide variety of forms.

The program employs six more variables to define the transformation matrices $\left(t_{x}, t_{y}, t_{z}, r_{x}, r_{y}, r_{z}\right)$ and some additional global variables such as the increment of value $t$, and the line length, which can either be fixed or modified by the user. These allow precise control of the relation between surfaces. Once the surfaces are assembled together, their intersections can be computed based on the properties of the rulings.

\subsection{REPRESENTATION OF REAL BUILDINGS}

The performance and efficiency of the system was tested by constructing concise, parametric descriptions for a series of existing structures, ranging in form from Calatrava's Bodegas Ysios and Bofill's El Prat Control Tower to Mies' Seagram Building.

The values of the parameters are presented in $13 \times \mathrm{n}$ matrices, with the exception of the hyperbolic paraboloid assembly. The matrices vary in size: they are $13 \times n$ where $n$ is the number of surfaces. Active parameters of each surface take a real or integer number value, otherwise they are set to 0 .

The traffic control tower of El Prat combines cylindrical and conical surfaces with a hyperboloid. Values of the matrix are presented in more detail in Table 3 . The effect of changing a single value in the matrix is demonstrated in Figure 2.
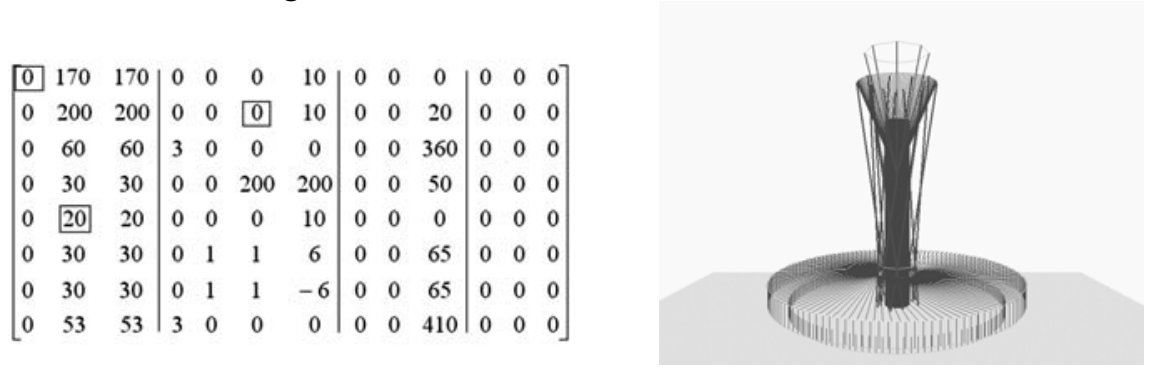

Figure 1. Traffic Control Tower-El Prat
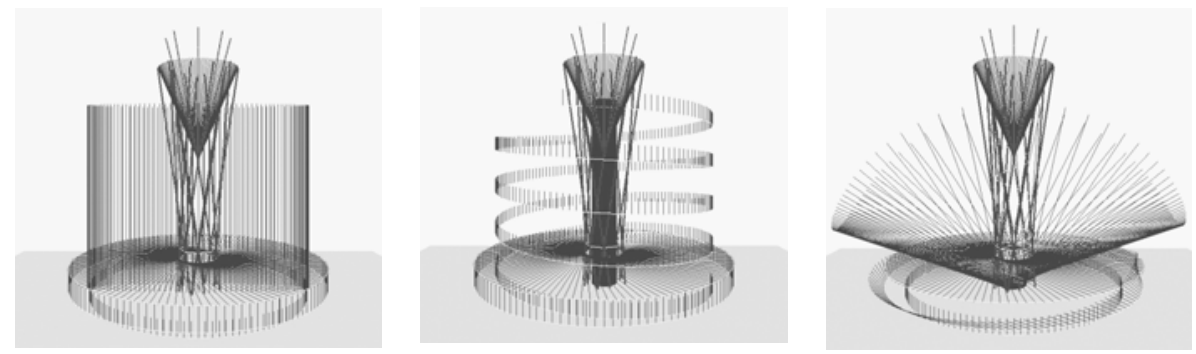

Figure 2. Variations of form by changing a value in the matrix 


\begin{tabular}{|c|c|c|c|c|}
\hline ROW & $\begin{array}{l}\text { BASE CURVE } \\
\text { parameters } \\
\text { trig. function }\end{array}$ & $\begin{array}{l}\text { DIRECTOR CURVE } \\
\text { parameters } \\
\text { trig. function }\end{array}$ & $\begin{array}{l}\text { RULED } \\
\text { SURFACE }\end{array}$ & $\begin{array}{l}\text { TRANSLATION } \\
\text { axis } \\
\text { units }\end{array}$ \\
\hline \multirow[t]{3}{*}{1} & $0,170,170$ & $0,0,0,10$ & & $0,0,0$ \\
\hline & {$[0,170 \cos t, 170 \sin t]$} & {$[0,0,10 \mathrm{t}]$} & & - \\
\hline & circle & line & & - \\
\hline \multirow[t]{3}{*}{2} & $0,200,200$ & $0,0,0,10$ & & $0,0,20$ \\
\hline & {$[0,200 \cos t, 200 \sin t]$} & {$[0,0,10 \mathrm{t}]$} & & Z \\
\hline & circle & line & & 20 \\
\hline \multirow[t]{3}{*}{3} & $0,60,60$ & $3,0,0,0$ & & $0,0,360$ \\
\hline & {$[0,60 \cos t, 60 \sin t]$} & {$[3 \mathrm{t}, 0,0]$} & & Z \\
\hline & circle & line & & 360 \\
\hline \multirow[t]{3}{*}{4} & $0,30,30$ & $0,0,200,200$ & & $0,0,50$ \\
\hline & {$[0,30 \cos t, 30 \sin t]$} & {$[200 \operatorname{cost}, 200 \operatorname{sint}, 0]$} & & $\mathrm{Z}$ \\
\hline & circle & circle & & 50 \\
\hline \multirow[t]{3}{*}{5} & $0,20,20$ & $0,0,0,10$ & & $0,0,0$ \\
\hline & {$[0,20 \cos t, 20 \sin t]$} & {$[0,0,10 \mathrm{t}]$} & & - \\
\hline & circle & line & & - \\
\hline \multirow[t]{3}{*}{6} & $0,30,30$ & $0,1,1,6$ & & $0,0,65$ \\
\hline & {$[0,30 \cos t, 30 \sin t]$} & {$[\cos t, \sin t, 6 t]$} & & $\mathrm{Z}$ \\
\hline & circle & helix & & 65 \\
\hline \multirow[t]{3}{*}{7} & $0,30,30$ & $0,1,1,-6$ & & $0,0,65$ \\
\hline & {$[0,30 \cos t, 30 \sin t]$} & {$[\cos t, \sin t,-6 t]$} & & Z \\
\hline & circle & helix & & 65 \\
\hline \multirow[t]{3}{*}{8} & $0,53,53$ & $3,0,0,0$ & \multirow{3}{*}{$\mathbb{V}$} & $0,0,410$ \\
\hline & {$[0,53 \cos t, 53 \sin t]$} & {$[3 \mathrm{t}, 0,0]$} & & Z \\
\hline & circle & line & & 410 \\
\hline
\end{tabular}

TABLE 3. Values of El Prat-matrix in detail 
In the case of Bodegas Ysios, the load bearing walls trace a sinusoidal shape and the roof is a ruled surface wave, which combines concave and convex surfaces as it evolves along the longitudinal axis.
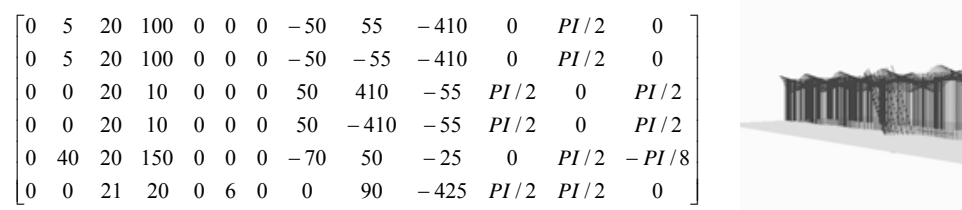

Figure 3. Bodegas Ysios

Palace of Assembly is a more complex composition of different surfaces: hyperboloid, planes, cylindrical surface in addition to a pyramid.

$\left[\begin{array}{ccccccccccccc}45 & 45 & 0 & 0 & 2 & 2 & 5 & 0 & 0 & 180 & 0 & 0 & 0 \\ 0 & 0 & 20 & 10 & 0 & 0 & 0 & 0 & 170 & 0 & 0 & 0 & 0 \\ 0 & 0 & 20 & 10 & 0 & 0 & 0 & 0 & -170 & 0 & 0 & 0 & P I / 2 \\ 0 & 0 & 20 & 10 & 0 & 0 & 0 & 0 & -170 & 0 & 0 & 0 & 0 \\ 0 & 0 & 20 & 10 & 0 & 0 & 0 & 0 & 170 & 0 & 0 & 0 & P I / 2 \\ 170 & 0 & 0 & 0 & 0 & 0 & 40 & 0 & 157 & 0 & P I / 2 & 0 & 0 \\ 0 & 0 & 20 & 10 & 0 & 0 & 0 & -240 & 145 & 0 & 0 & 0 & 0 \\ 0 & 0 & 20 & 10 & 0 & 0 & 0 & -240 & 95 & 0 & 0 & 0 & 0 \\ 0 & 0 & 20 & 10 & 0 & 0 & 0 & -240 & 45 & 0 & 0 & 0 & 0 \\ 0 & 0 & 20 & 10 & 0 & 0 & 0 & -240 & -5 & 0 & 0 & 0 & 0 \\ 0 & 0 & 20 & 10 & 0 & 0 & 0 & -240 & -55 & 0 & 0 & 0 & 0 \\ 0 & 0 & 20 & 10 & 0 & 0 & 0 & -240 & -105 & 0 & 0 & 0 & 0 \\ 0 & 0 & 20 & 10 & 0 & 0 & 0 & -240 & -155 & 0 & 0 & 0 & 0 \\ 30 & 20 & 0 & 0 & 0 & 0 & 10 & -240 & -100 & 0 & -P I / 2 & 0 & 0 \\ 0 & 0 & 20 & 10 & 0 & 0 & 0 & 0 & -120 & 110 & 0 & -P I / 6 & P I / 2 \\ 0 & 0 & 20 & 10 & 0 & 0 & 0 & 0 & -120 & 110 & -P I / 6 & 0 & 0 \\ 0 & 0 & 20 & 10 & 0 & 0 & 0 & 0 & 120 & 110 & 0 & P I / 6 & P I / 2 \\ 0 & 0 & 20 & 10 & 0 & 0 & 0 & 0 & 120 & 110 & P I / 6 & 0 & 0\end{array}\right]$

Figure 4. Palace of Assembly

Los Manantiales provides an illustrative sample of an assembly of hyperbolic paraboloids rotated around a central point. It was selected to test the extended version of the program.
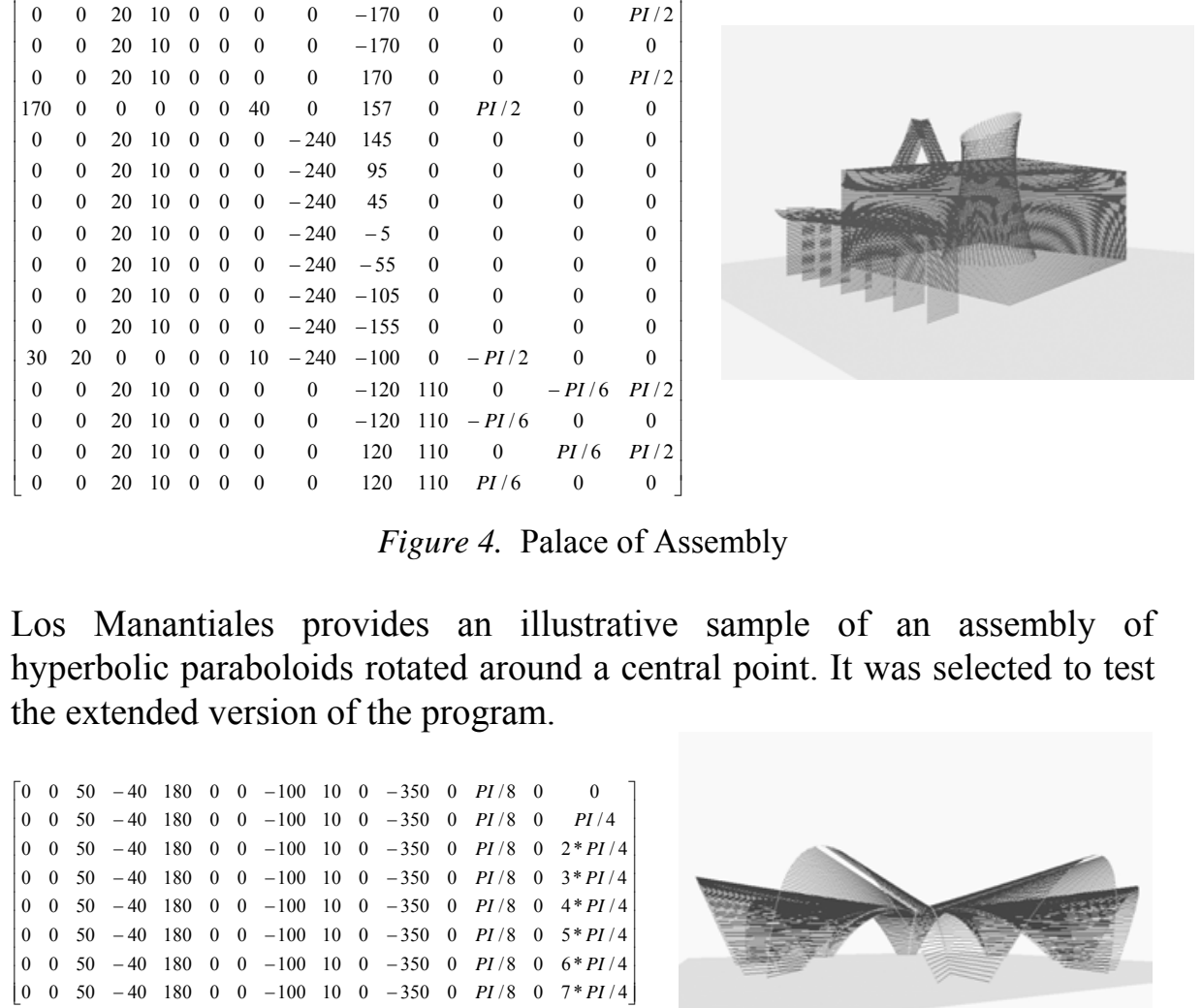
The Seagram Building is composed of planar surfaces. It was selected for a comparison to the representation proposed by L.March.
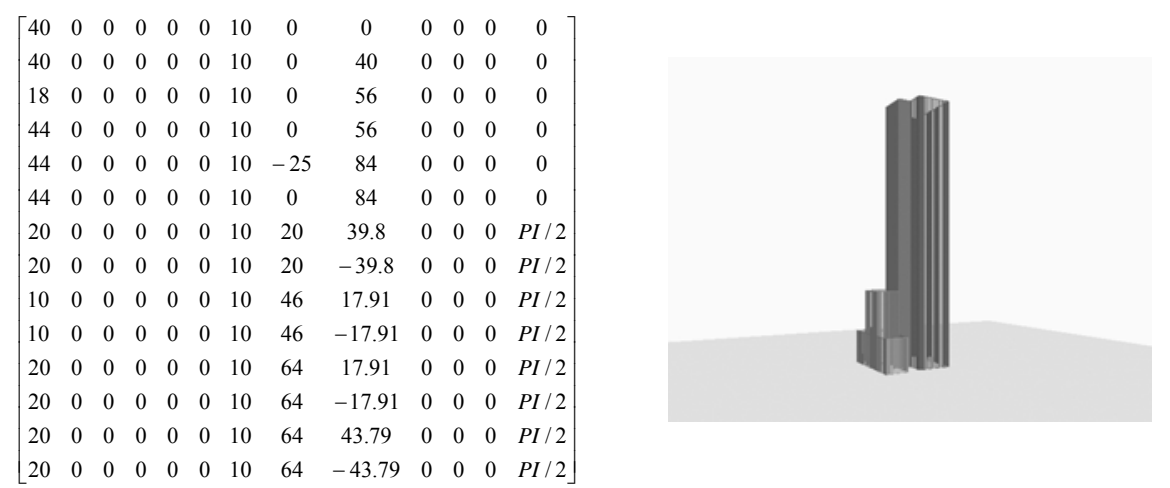

Figure 6. Seagram Building

\section{Discussion}

\subsection{GENERAL CHARACTERISTICS OF THE SYSTEM}

The parametric representation provides significant information in the matrix of values. Looking at the matrix, information can be instantly acquired about the type of surfaces that compose the structure (planar or curvilinear), the number of different types, various height levels, existence of repeated elements, relation between the locations of elements etc. Also some values, possibly signifying common features, can be directly read.

By expressing a structure as a series of values that correspond to a special configuration of elements, the designer can instantly create multiple variations of form presented as three-dimensional models. The user interacts by altering values. The model can be modified a limitless amount of times while always maintaining relations between parts. Embedding rules and constrains in the representation, in the form of parameters transforms the otherwise "passive" representation into an active "one" (Kalay 2004). It has been observed from the results that small changes in values can lead to unexpected changes of form. Thus experimenting with random values can lead to interesting creations that may provoke the designer's creativity.

The use of a limited number of variables renders the representation highly flexible. Elements can be assembled in an unlimited number of ways. Moreover it is efficient in terms of speed and simplicity. Embedding rules and relations between elements of the system makes it very easy to understand, manipulate and use. With no special effort a three-dimensional model can be produced simply by entering a number of values. 


\subsection{LIMITATIONS}

The intention of using minimal data restricts the application of the parametric representation. It is not applicable to structures that are composed either of ruled surfaces swept along a free form curve, such as DeCOi's Paramorph, or of free form surfaces, such as the Guggenheim Museum in Bilbao.

\subsection{FURTHER WORK}

Apart from further development of the representation to include free form curves, it can be integrated with 3D CAD/modelling software as an exploratory generative design tool. Another general development can be the implementation of constraints that connect one surface to the other such as parallelism, perpendicularity and tangency. If the representation is used as a generative design method, constraints such as surface continuity would be useful to ensure that all designs make sense geometrically, or to reach objectives such as structural stability. Constraints can also be specified as conditional relations. Incorporating conditional expressions might extend the interest of the method.

The parametric representation may also be implemented in search techniques. The proposed representation would be integrated in a genetic algorithm by mapping the parameters that describe a surface to a genotype, the alleles of which would correspond to the set of values. An initial set of rules and constraints responding to a given problem and specification of fitness criteria is required, by which a fitness test would evaluate how close the values are to a given example or criterion. Such a process could be used to find a rational ruled surface definition resembling any given form, as in the building representations in section 4 , and serve as a point of departure for further design exploration. Alternatively, an analysis of solar shading, structural performance or other properties could be performed on the model and the form optimised to solve a specific design problem.

\section{Conclusions}

Creating a parametric representation involves choosing the right parameters and establishing connections between them. In this case it has been based on relations between elements that are both simple and economic: the variations produced by the transformation of the helix. This economy of parameters results in a model that is powerful in that it requires very little numeric data to describe a large range of possible forms.

The number of parameters needed (the fewer the better) and the range of forms capable of being represented (the more the better) are the evaluation criteria for the method. In terms of representation, this minimises 
redundancy in the data required. In terms of search (e.g. genetic algorithm), it reduces the dimensionality of the search space and therefore simplifies the process.

The rich spectrum of produced forms, including planar and a significant number of curvilinear forms, do describe the geometry of a large number of buildings and because of the achieved parameterisation of the various generating curves in a single model, the number of parameters needed is reduced. The more familiar method of joining two boundary curves by line segments has the advantage of allowing any freeform ruled surfaces to be defined, but the majority of curves used in actual buildings are rational curves defined by the proposed method, and for these the parameterisation is minimal.

For buildings consisting of ruled surfaces, a significant amount of information about the form can be encoded in the matrix of values. Having a limited number of variables increases flexibility of representation.

Using surfaces composed of straight lines as building blocks of the system may simplify static calculations as well as the actual construction. The model implicitly offers indications of the structure and its behaviour.

Minimal encoding based on numerical parameters could benefit greatly from employing constraints or links between design elements, something that can be easily achieved in this model because it is mathematically explicit. Constraints are a part of implementation to follow depending on how the model is to be used.

The resulting implementation allows an interactive manipulation of the model by changing parameters. Multiple variations of the model can be rapidly produced so that design exploration is performed by selection of optimal variations. The procedure is simple, developed for a limited number of parameters, user-friendly and efficient in terms of speed. Apart from the integration of the representation in 3D modelling software, encoding structures in this way makes possible its integration in evolutionary design techniques, such as genetic algorithms.

It has been demonstrated how a simple parametric system based on relations of elements and using limited amount of data can be developed as a useful design tool. It can sufficiently represent a wide variety of forms efficiently and with few variables.

\section{Acknowledgements}

The authors would like to thank Chiron Mottram for his assistance in the development of the Processing program and his comments. 


\section{References}

Bentley, P: 1999, An Introduction to Evolutionary Design by Computers, in P Bentley (ed.), Evolutionary design by Computers, Morgan Kaufmann, San Francisco.

Bentley, P: 1999(a), From Coffee Tables to Hospitals: Generic Evolutionary Design, in P Bentley (ed.), Evolutionary design by Computers, Morgan Kaufmann, San Francisco.

Burry, M: 1993, Expiatory Church of the Sagrada Família, Phaidon Press, London.

Burry, M: 2003, Between Intuition and Process: Parametric Design and Rapid Prototyping, in B. Kolarevic (ed.), Architecture in the Digital Age- Design and Manufacturing, Spon Press, New York.

Burry, M, Burry J, Dunlop, GM and Maher A: Drawing Together Euclidean and Topological Threads, presented at SIRC 2001.

Coates, P, Broughton, T and Jackson, H: 1999, Exploring Three-Dimensional Design Worlds using Lindenmayer Systems and Genetic Programming, in P. Bentley (ed.) Evolutionary design by Computers, Morgan Kaufmann, San Francisco.

DeCOi: 2000, Technological Latency: from Autoplastic to Alloplastic, Digital Creativity, 11 (3): 131-143.

English, EC: 2005, Vladimir Shukhov and the Invention of Hyperboloid Structures, Metropolis \& Beyond: Proceedings of the 2005 Structures Congress and the 2005 Forensic Engineering Symposium, New York.

Frazer, J: 1995, An Evolutionary Architecture, Architectural Association Publications London.

Jackson, H: 2002, Toward a Symbiotic Coevolutionary Approach to Architecture, in P Bentley and W Corne (eds), Creative evolutionary systems, Morgan Kaufmann, San Francisco.

Kalay, YE: 2004, Architecture's New Media : Principles, Theories, and Methods of Computer-Aided Design, MIT Press, Cambridge.

Kühnel, W: 2002, Differential Geometry : Curves - Surfaces - Manifolds, AMS, Rhode Island.

March, L: 1972, A Boolean description of a class of built forms, Cambridge University Press, Cambridge.

March, L and Steadman, P: 1971, The Geometry of the Environment, R.I.B.A Publications, London.

O'Neill, B: 1966, Elementary Differential Geometry, Academic Press New York.

Steadman, P: 1998, Sketch for an archetypal building, Environment and Planning B: Planning and Design, 25th Anniversary Issue, pp. 92-105.

Steadman, P: 2001, Binary encoding of a Class of Rectangular Built-Forms, Proceedings, 3rd International Space Syntax Symposium, Atlanta.

Steadman, P and Waddoups, L: 2000, A Catalogue of Built Forms, using a Binary Representation. Proceedings, 5th International Conference on Design and Decision Support Systems in Architecture, Nijkerk, The Netherlands, pp. 353-373.

Todd, S and Latham, W: 1999, The Mutation and Growth of Art by Computers, in P Bentley (ed.), Evolutionary design by Computers, Morgan Kaufmann, San Francisco.

Rosenman, M and Gero, J: 1999, Evolving Designs by Generating Useful Complex Gene Structures, in P Bentley (ed.), Evolutionary design by Computers, Morgan Kaufmann, San Francisco. 